\title{
ANÁLISE NÃO LINEAR FÍSICA E GEOMÉTRICA EM ENSAIO DE COMPRESSÃO PARALELA: CASO DE ESTUDO NA MADEIRA MARUPÁ
}

\author{
PHYSICAL AND GEOMETRIC NONLINEAR ANALYSIS IN A PARALLEL \\ COMPRESSION TEST: A CASE STUDY IN MARUPÁ WOOD
}

\section{RESUMO}

Este artigo apresenta uma análise não linear física e geométrica de uma amostra de madeira da espécie Simarouba amara Aubl., conhecida popularmente por "Marupá". Para tal foram efetivados ensaios experimentais para obter a relação constitutiva dessa madeira em testes de compressão paralela às fibras. Os resultados experimentais foram utilizados para calibrar o modelo numérico considerado. Com relação ao modelo numérico, empregou-se a formulação do Método dos Elementos Finitos Posicional acoplado ao modelo de dano FLHB, os quais levam em consideração a não-linearidade geométrica e física, respectivamente. Dessa forma, esse acoplamento descreveu com precisão todos os trechos da curva experimental (encruamento, amolecimento e ruptura) para o ensaio de compressão paralela às fibras de madeira.

Palavras-chave: Compressão paralela às fibras. Método dos Elementos Finitos Posicional. Madeira.

\section{ABSTRACT}

This paper presents a physical and geometric nonlinear analysis of a wood specimen of the species Simarouba amara Aubl, popularly known as Marupá. Compression parallel to the grain mechanical test was performed for obtaining the constitutive relation. The experimental results were used to calibrate the considered model of numerical analysis. The Positional Finite Element Method with the damage model FLHB were considered for the numerical analysis, which consider both physical and

1 Doutor em Engenharia Civil (Engenharia de Estruturas) pela Universidade de São Paulo (USP). E-mail: tulio-raunyr@usp.br 2 Graduada em Engenharia Civil pela Universidade Brasil. E-mail: jehlinares@hotmail.com 3 Doutor em Ciências e Engenharia de Materiais pela Universidade de São Paulo (USP). E-mail: tiago.hendrigo@gmail.com 4 Graduado em Engenharia Civil pela Universidade Brasil. E-mail: lucas-factor@hotmail.com

5 Graduando em Engenharia Civil pelo Centro Universitário de João Pessoa (UNIPÊ). E-mail: kaliel.gomes@hotmail.com

\section{INTER SCIENTIA}


geometric nonlinearities, respectively. Based on the results, this approach described accurately the whole experimental curve (hardening, softening and rupture) for the compression parallel to the grain test.

Keywords: Compression parallel to the grain. Positional Finite Element Method. Wood.

\section{INTRODUÇÃO}

A madeira é utilizada em diversos setores industriais e na construção civil apresentando menor impacto ambiental com a sua utilização (ALMEIDA et al., 2017). A madeira quando comparada com outros materiais de construção, proporciona uma boa relação resistência/peso, além de conter outros itens convenientes como facilidade de fabricação de inúmeros produtos industrializados, isolamento acústico e térmico (PFEIL, 2003).

Mesmo sendo um material inflamável, a madeira apresenta um excelente desempenho sobre altas temperaturas quando comparada a outros materiais convencionais, pois a mesma quando exposta às chamas, apenas sua superfície é degradada, queimando lentamente, o que evita colapso, ao contrário do aço (MARTINS, 2016).

Os elementos estruturais de madeira podem ser utilizados de forma provisória ou definitiva. Os elementos classificados como provisórios são os utilizados durante o andamento da obra, como por exemplo as formas e cibramentos. Por outro lado, os elementos definitivos são os estruturais (pilares, vigas e treliças), bem como os componentes de edificação (pisos, esquadrias e revestimentos) (CALIL JUNIOR et al., 2003).

○ Marupá (Simarouba amara Aubl.) apresenta propriedades apreciadas, sendo muito utilizada na construção civil. Ela apresenta leveza, facilidade de manuseio e uma excelente resistência ao ataque de insetos (AZEVEDO et al., 2009). Além disso, ela exibe uma secagem muito eficaz, podendo ser feita ao ar livre desde que essa esteja coberta e bem empilhada, para se evitar danos no material (IPT, 1990). A madeira dessa espécie é classificada como C20 de acordo com a ABNT NBR 7190:1997, proporcionado valor característico da resistência à compressão paralela às fibras maior ou igual a $20 \mathrm{MPa}$.

Os comportamentos linear e não linear estão sempre presentes nos elementos estruturais. Esses podem ser diferenciados através do diagrama Força vs. Deslocamento. Neste diagrama, o comportamento linear é graficamente definido através de uma reta, enquanto o não linear é representado por uma curva (PROENÇA, 2016). 
A não linearidade pode ser geométrica, física ou de contato. A não linearidade geométrica corresponde aos efeitos de variação de posição da estrutura. Neste sentido, as condições de equilíbrio da estrutura são avaliadas na configuração atual (BONET et al., 2000). Com relação a não linearidade física, esta ocorre quando a relação constitutiva do material é não linear, ou seja, a tensão não depende linearmente da deformação (CORRÊA, 2015). Por último, a não linearidade de contato diz respeito ao relaxamento das restrições de invariabilidade das condições de contorno em força e/ou deslocamento (PROENÇA, 2016).

Como tradicionalmente as estruturas de madeira são dimensionadas no regime linear, diante de situações extremas, essas estruturas podem falhar uma vez que não são consideradas a resposta do material e análise na configuração atual. Portanto, a análise não linear é fundamental para avaliar trajetória de equilíbrio dessas estruturas.

\section{OBJETIVOS}

Este trabalho objetiva avaliar e discutir sobre a análise não linear física e geométrica de uma amostra de madeira da espécie Simarouba amara Aubl sujeita ao teste de compressão paralela às fibras.

\section{MATERIAIS E MÉTODOS}

Dois corpos de prova de madeira isentos de defeitos (CP-1 e CP-2), com dimensões nominais $21,8 \mathrm{~mm} \times 21,9 \mathrm{~mm} \times 60 \mathrm{~mm}$ (maior dimensão na direção das fibras), foram ensaiados à compressão paralela às fibras de acordo com o documento normativo ABNT NBR 7190:1997. Tal ensaio foi realizado na máquina servo-hidráulica universal, marca INSTRON, modelo 8506, com capacidade para $1500 \mathrm{kN}$.

No ensaio de compressão paralela às fibras, os extensômetros (Strain Gages) foram colocados de acordo com a direção da aplicação da carga, como mostra a Figura 1. Foi empregado também um transdutor de deslocamento (LVDT), a fim de obter um comparativo entre as medidas via extensômetros com o LVDT. 
Figura 1 - Montagem do experimento para o ensaio de compressão paralela às fibras.

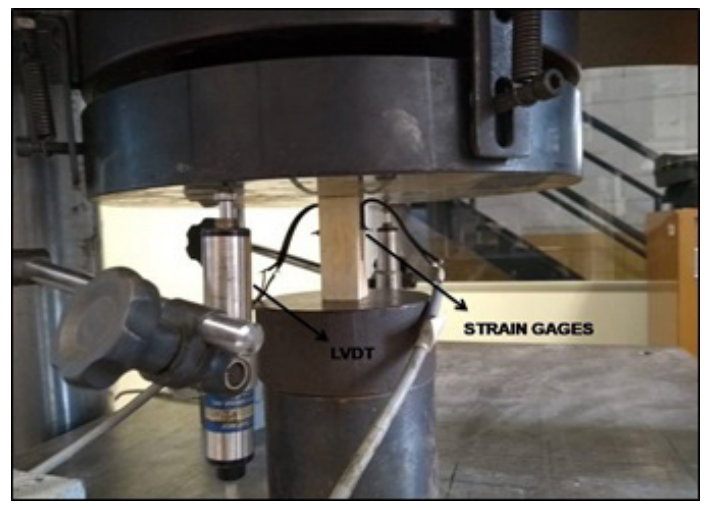

Fonte: Autores.

O módulo de elasticidade foi obtido no trecho linear do diagrama Tensão vs. Deformação conforme as recomendações do documento normativo brasileiro ABNT NBR 7190:1997.

As análises numéricas foram realizadas via método dos elementos finitos posicional (MEFP), no qual a não linearidade geométrica já é levada em consideração em suas deduções (BONET et al., 2000; CODA e GRECO, 2004). Para tal, utilizou-se a formulação do MEFP para o elemento de treliça apresentada por Felipe (2019). As equações e o algoritmo para a implementação numérica podem ser vistos no trabalho desse autor. Neste trabalho, empregou-se o programa computacional desenvolvido por Felipe (2019).

Com relação a não-linearidade física, adotou-se o modelo de dano FLHB proposto por Felipe et al. (2019) acoplado ao MEFP. Esse modelo captura a degradação mecânica por meio da variação de porosidade no material. As equações desse modelo e a validação para onze materiais diferentes são apresentados no trabalho desses autores. Esse modelo foi acoplado ao programa computacional desenvolvido por Felipe (2019).

\section{RESULTADOS E DISCUSSÕES}

Realizando-se os ensaios mecânicos, verificou-se que o CP-1 rompeu por cisalhamento, como mostra Figura 2. Nota-se que a inclinação do plano de ruptura resultou aproximadamente $45^{\circ}$ (direção da tensão de cisalhamento máxima). 
Figura 2 - Modo de ruptura do CP-1.

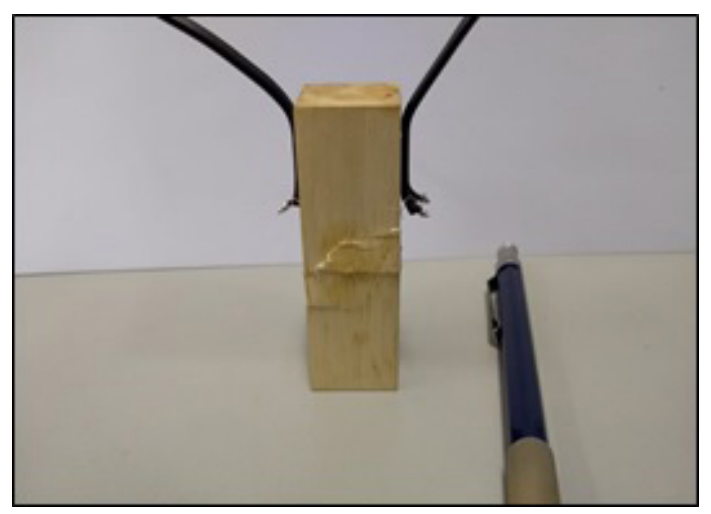

Fonte: Autores.

Por outro lado, CP-2 rompeu por esmagamento da base, conforme ilustrado na Figura 3. Isso ocorreu devido à concentração de tensão na base, a qual indica que a superfície não estava perfeitamente plana. Sendo assim, apenas o CP-1 foi considerado para a análise numérica.

Figura 3 - Modo de ruptura do CP-2.

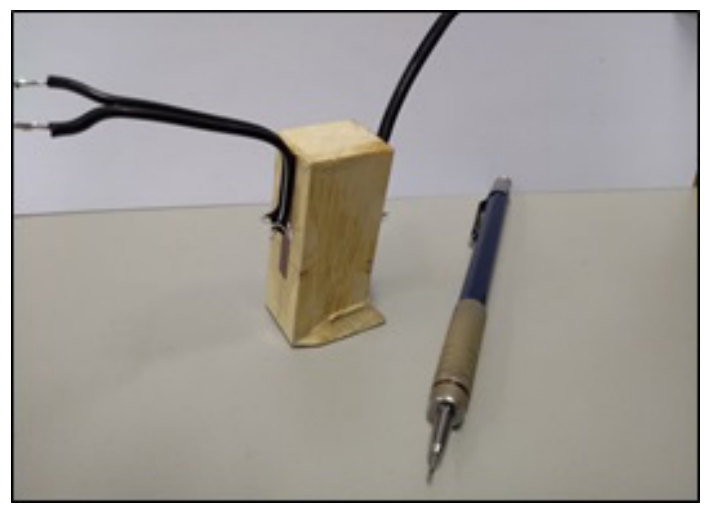

Fonte: Autores.

As curvas Tensão vs. Deformação resultantes do ensaio de compressão paralela às fibras do CP-1 estão apresentadas na Figura 4, utilizando os dados do LVDT e dos Strain gages. Observa-se que a curva do LVDT possui uma menor rigidez devido à conformação inicial do material. Já a curva obtida através dos extensômetros apresentou-se mais rígida. Neste caso, como apresenta-se duas medidas de deformação, há necessidade de realizar-se uma correção dos dados, e essa correção é determinada para o LVDT por meio da Equação 1 (OSORIO, BAIRÁN e MARÍ, 2013). 
Figura 4 - Curva Tensão vs. Deformação para o ensaio de compressão paralela às fibras.

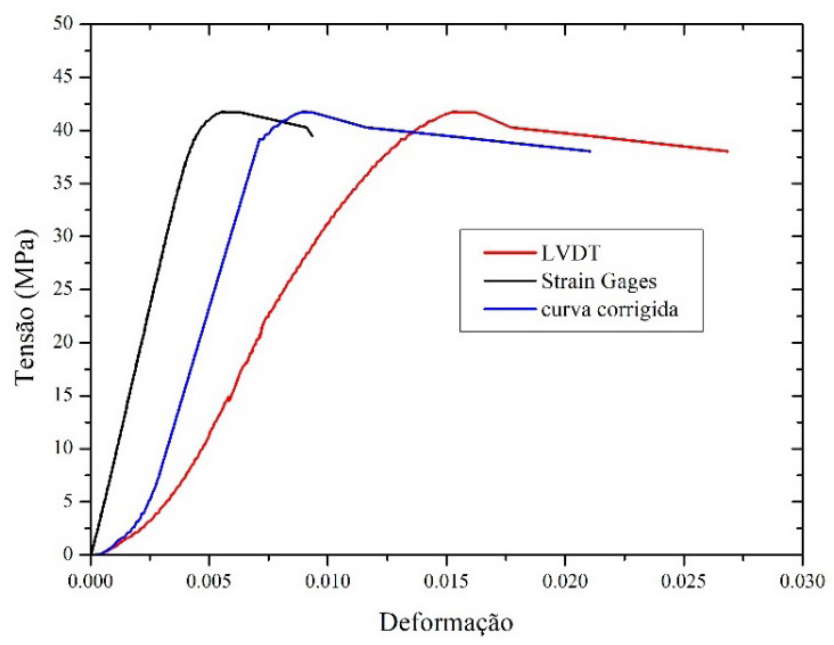

Fonte: Autores.

$$
\varepsilon_{i}=\varepsilon_{i, L V D T}-\left(\frac{E_{\text {gages }}-E_{L V D T}}{E_{\text {gages }} \cdot E_{L V D T}}\right) \cdot \sigma_{i}
$$

Na equação 1, $\varepsilon_{i}$ é a deformação corrigida para o nível de tensão $\sigma_{i}{ }^{\prime} \varepsilon_{i, L V D T}$ é a deformação obtida através do LVDT; $E_{\text {gages }}$ é o módulo de elasticidade calculado para a curva via extensômetros; $E_{L V D T}$ é o módulo de elasticidade determinado a partir do LVDT. Os valores dos módulos de elasticidade foram determinados de acordo com a ABNT NBR 7190:1997.

De acordo com a Figura 4, no início da curva do LVDT ocorre uma deformação residual. Isto ocorre porque existe acomodação do dispositivo de ensaio. A Figura 4 apresenta a curva corrigida do ensaio de compressão paralela às fibras para o CP-1.

Para a análise numérica utilizou-se um elemento finito de treliça com as propriedades físicas e geométricas do corpo de prova CP-1 obtidos no ensaio mecânico realizado, conforme apresentado no Quadro 1. As condições de contorno podem ser vistas na Figura 5. Quatrocentos passos de cargas foram usados, com incremento de $0,00315 \mathrm{~mm}$. O critério de convergência foi em posição para uma tolerância de $10^{-6}$. A definição de cada parâmetro $\varepsilon_{d, 0^{\prime}} a_{p, 1^{\prime}} a_{p, 2^{\prime}} a_{p, 3} \in D_{c r i t}$ do modelo de dano FLHB pode vista em Felipe et al. (2019). 
Quadro 1 - Dados físicos e geométricos do CP-1.

\begin{tabular}{c|c|c|c|c|c|c|c|c}
\hline$I_{0}(\mathbf{m m})$ & $A_{0}\left(\mathrm{~mm}^{2}\right)$ & $E(\mathbf{M P a})$ & $\sigma_{y}(\mathbf{M P a})$ & $\varepsilon_{d, 0}$ & $a_{p, 1}$ & $a_{p, 2}$ & $a_{p, 3}$ & $D_{c r i t}$ \\
\hline 60,00 & 477,40 & 9513 & 5,80 & $1,51 \cdot 10^{-3}$ & $-3255,9$ & 34,884 & $-0,0014$ & 0,088 \\
\hline
\end{tabular}

Fonte: Autores.

Figura 5 - Propriedades geométricas e condição de contorno para o modelo numérico.

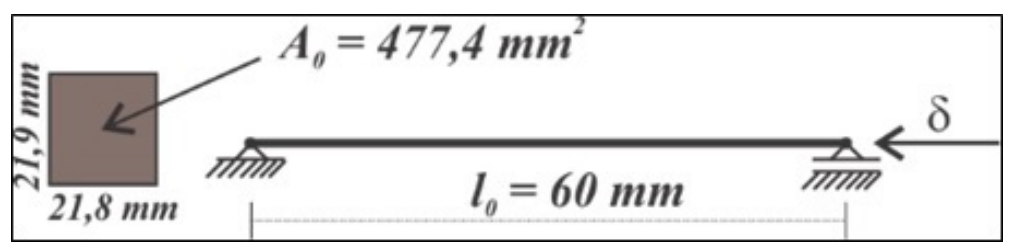

Fonte: Autores.

A Figura 6 apresenta resultado da análise numérica confrontada com os resultados experimentais, mostrando a compatibilidade da resposta numérica. Observa-se que o mecanismo de ruptura dessa madeira à compressão ocorreu com amolecimento em níveis de deformações moderadas. Devido à orientação das fibras, a madeira é um material anisotrópico. Este efeito não é avaliado pelo modelo FLHB. Todavia, como o ensaio foi executado com carga aplicada paralela às fibras, nesta direção não há variação das propriedades elásticas. Consequentemente, o modelo FLHB pode ser utilizado para a avalição da madeira, assumindo-se que na direção considerada as propriedades elásticas são constantes.

Figura 6 - Ensaio de compressão paralela às fibras: resposta numérica em comparação a resposta experimental: (a) relação constitutiva; (b) evolução do dano e (c) módulo tangente.

(a)

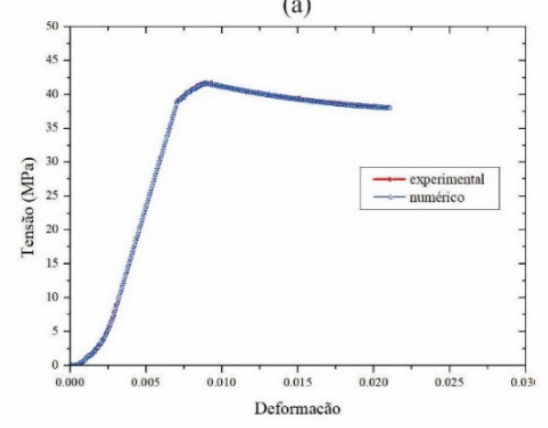

(b)

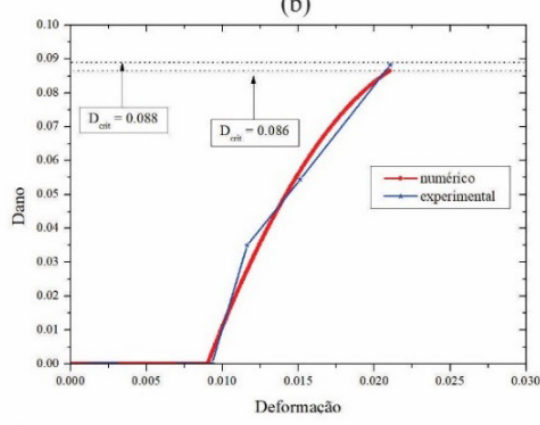

(c)

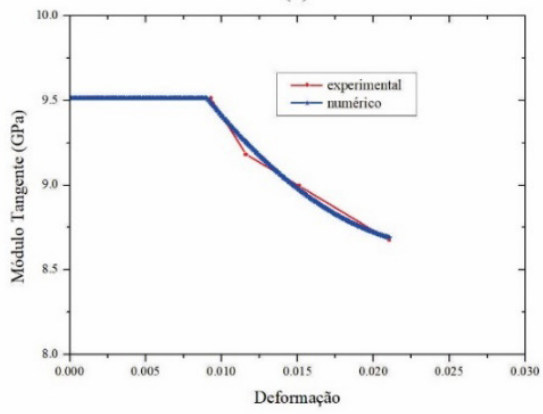

Fonte: Autores. 
Outrossim, a formulação MEFP acoplada ao modelo de dano FLHB descreve com precisão todos os trechos da curva experimental (encruamento, amolecimento e ruptura) para o ensaio de compressão paralela às fibras da madeira. Ademais, nota-se a boa acurácia dessa formulação na avaliação da evolução do dano e do módulo da tangente em comparação aos resultados obtidos experimentalmente.

\section{CONCLUSÕES}

De acordo com os resultados foi possível concluir que a planicidade da seção transversal do corpo de prova analisado pode influenciar no tipo de ruptura da madeira espécie Simarouba amara Aubl. Observou-se que a evolução do dano cresce até o dano crítico $\left(D_{\text {crit }}\right)$. Dessa forma, o módulo tangente obtido via curva numérica tende ao valor calculado pelo gráfico experimental, em conformidade com a Figura 6-(c). Por conseguinte, o modelo de dano FLHB não conduz singularidade na matriz Hessiana, implicando que na análise numérica não ocorre perda de convergência. Por fim, verificou-se a boa acurácia do acoplamento da formulação do MEFP com o modelo FLHB.

\section{REFERÊNCIAS}

ALMEIDA, T. H.; CHRISTOFORO, A. L.; LAHR, F. A. R. Study of Dimensional Stability of Brazilian Tropical Wood Species. Saarbrüken-Deutschland/Germany: Lambert Academic Publishing, v. 1, 2017.

ASSOCIAÇÃO BRASILEIRA DE NORMAS TÉCNICAS. NBR 7190. Projeto de estruturas de madeira. Rio de Janeiro, 1997.

AZEVEDO, I. M. G.; AlENCAR, R. M.; BARBOSA, A. P.; ALMEIDA, N. O. Estudo do crescimento e qualidades de muda de marupá (Simarouba amara) em viveiro. Amazonas, 2009.

BARKER, B.; OWEN, N. L. Identifying Softwoods and Hardwoods by Infrared Spectroscopy. Journal of Chemical Education, v. 76, n. 12, p. 1706-1709, Dez. 1999.

BONET, J.; WOOD, R. D.; MAHANEY, J.; HEYWOOD, P. Finite element analysis of air supported membrane structures. Computer methods in applied mechanics and engineering, v. 190, n. 5-7, p. 579-595, 2000.

BRITO, L. D. Patologia em Estruturas de Madeira: Metodologia de Inspeção e Técnicas de Reabilitação. São Carlos, 2014.

CALIL JR, C.; LAHR, F. A. R.; DIAS, A. A. Dimensionamento de elementos estruturais de madeira. 1. ed. Barveri: Manole, 2003. 
CODA, H. B.; GRECO, M. A simple FEM formulation for large deflection 2D frame analysis based on position description. Computer methods in applied mechanics and engineering, v. 193, n. 33-35, p. 3541-3557, 2004.

CORRÊA, M. R. Introdução ao comportamento não linear de estruturas. [S.L], 2015.

FELIPE, T. R. C. Análise mecânica e probabilística de estruturas treliçadas sujeitas ao colapso progressivo. São Carlos, 2019.

FELIPE, T. R. C.; LEONEL, E. D.; HAACH, V. G.; BECK, A. T. A comprehensive ductile damage model for 3D truss structures. International Journal of Non-Linear Mechanics, v. 112, p. 13-24, 2019.

IPT. Instituto de Pesquisas Tecnológicas. Informações sobre a madeira. Jankowsky, 1990.

MARTINS, G. C. A. Análise Numérica e Experimental de vigas de Madeira Laminada Colada em Situação de Incêndio. São Carlos, 2016.

NOGUEIRA, R. S. Proposta de um método de ensaio para controle de qualidade na produção de elementos estruturais de MLC e de LVL. São Carlos, 2017.

OSORIO, Edison; BAIRÁN, Jesuús M.; MARÍ, Antonio R. Lateral behavior of concrete under uniaxial compressive cyclic loading. Materials and Structures, v. 46, p. 709724, 2013.

PFEIL, W; PFEIL, M. Estruturas de Madeira. Rio de Janeiro, 2003.

PROENÇA, S. P. B. Análise não - linear de estruturas. São Carlos, 2016.

REMADE. Madeiras Brasileiras e Exóticas. Paraná, 2018.

RUBERT, J. B. Estudo do desempenho de algoritmos numéricos na solução de sistemas não lineares de estruturas formadas por barras de treliça. São Carlos, 1993.

ZENID, G. J. Identificação e Grupamento das Madeiras Serradas Empregadas na Construção Civil Habitacional na Cidade de São Paulo. Piracicaba, 1997. 